\title{
The atmospheric chemistry of the warm Neptune GJ 3470b: Influence of metallicity and temperature on the $\mathrm{CH}_{4} / \mathrm{CO}$ ratio
}

\author{
Olivia Venot ${ }^{1}$, Marcelino Agúndez ${ }^{2,3}$, Franck Selsis $^{2,3}$, Marcell Tessenyi $^{4}$, and Nicolas Iro ${ }^{5}$ \\ ${ }^{1}$ Instituut voor Sterrenkunde, Katholieke Universiteit Leuven, Celestijnenlaan 200D, 3001 Leuven, Belgium \\ 2 Univ. Bordeaux, LAB, UMR 5804, 33270 Floirac, France \\ 3 CNRS, LAB, UMR 5804, 33270 Floirac, France \\ ${ }^{4}$ University College London, Department of Physics and Astronomy, Gower Street, London WC1E 6BT, UK \\ 5 Theoretical Meteorology group, Klimacampus, University of Hamburg, Grindelberg 5, 20144 Hamburg, Germany \\ e-mail: olivia.venot@ster.kuleuven.be
}

Received 14 August 2013 / Accepted 18 December 2013

\begin{abstract}
Context. Current observation techniques are able to probe the atmosphere of some giant exoplanets and get some clues about their atmospheric composition. However, the chemical compositions derived from observations are not fully understood. For instance, the $\mathrm{CH}_{4} / \mathrm{CO}$ abundance ratio is often inferred to be different from the value that has been predicted by chemical models. Recently, the warm Neptune GJ 3470b has been discovered, and because of its close distance from us and high transit depth, it is a very promising candidate for follow-up characterisation of its atmosphere.

Aims. We study the atmospheric composition of GJ 3470b to compare to the current observations of this planet and to prepare for future ones but also to understand the chemical composition of warm (sub-)Neptunes as a typical case study. The metallicity of such atmospheres is totally uncertain and are likely to vary to values up to $100 \times$ solar. We explore the space of unknown parameters to predict the range of possible atmospheric compositions.

Methods. We use a one-dimensional chemical code to compute a grid of models with various thermal profiles, metallicities, eddy diffusion coefficient profiles, and stellar UV incident fluxes. Thanks to a radiative transfer code, we then compute the corresponding emission and transmission spectra of the planet and compare them with the observational data already published.

Results. Within the parameter space explored we find that methane is the major carbon-bearing species in most cases. We, however, find that for high metallicities with a sufficiently high temperature, the $\mathrm{CH}_{4} / \mathrm{CO}$ abundance ratio can become lower than unity, as suggested by some multiwavelength photometric observations of other warm (sub-)Neptunes, such as GJ 1214b and GJ 436b. As for the emission spectrum of GJ 3470b, brightness temperatures at infrared wavelengths may vary between 400 and $800 \mathrm{~K}$ depending on the thermal profile and metallicity.

Conclusions. Combined with a hot temperature profile, a substantial enrichment in heavy elements by a factor of $\geq 100$ with respect to the solar composition can shift the carbon balance in favour of carbon monoxide at the expense of methane. Nevertheless, current observations of this planet do not allow us yet to determine which model is more accurate.
\end{abstract}

Key words. planets and satellites: atmospheres - planets and satellites: composition - planets and satellites: individual: GJ 3470b astrochemistry

\section{Introduction}

In the past, multiwavelength observations of transiting exoplanets have been used to provide the first constraints on the chemical composition of exoplanet atmospheres. The identification of atmospheric constituents is currently restricted to gas giant planets with small orbital distances because of the large transit depth variations. Most efforts have concentrated on Jupitersized planets that orbit around solar-type stars: the so-called hot Jupiters. These planets are heavily irradiated by the nearby (early K-, G-, or late F-type) star, resulting in planetary equilibrium temperatures in excess of $1000 \mathrm{~K}$. Transmission and dayside emission spectra of hot Jupiters, such as HD 209458b and HD 189733b, have revealed the presence of molecules, such as $\mathrm{CO}, \mathrm{H}_{2} \mathrm{O}, \mathrm{CH}_{4}$, and $\mathrm{CO}_{2}$, in their atmospheres (Tinetti et al. 2007; Grillmair et al. 2008; Swain et al. 2008, 2009a,b; Madhusudhan \& Seager 2009), although contradictory conclusions among different studies are not rare. Chemical models of hot Jupiter atmospheres, which incorporate different degree processes such as thermochemical kinetics, vertical mixing, horizontal transport, and photochemistry (Line et al. 2010; Moses et al. 2011; Kopparapu et al. 2012; Venot et al. 2012; Agúndez et al. 2012), indicate that carbon monoxide and water vapour should be the major reservoirs of carbon and oxygen in this kind of hot hydrogen-helium dominated atmosphere, while methane and carbon dioxide would be less abundant.

Even more challenging, transit spectra have recently allowed us to characterise the atmosphere of the Neptune-sized planet GJ 436b and the mini Neptune or super-Earth GJ 1214b, which both orbit around M dwarf stars. Unlike Jupiter-sized planets, which have a very low occurrence rate around $\mathrm{M}$ dwarf stars (Johnson et al. 2007; Bonfils et al. 2013), (sub-)Neptune-sized planets are found around both solar-type and M dwarf stars, although they are more easily observed around the latter type of stars because of the higher planet-to-star contrast, which favour primary and secondary transit observations. These planets have at least a couple of interesting differences with respect to hot Jupiters. The first is because the host M dwarf star is smaller 


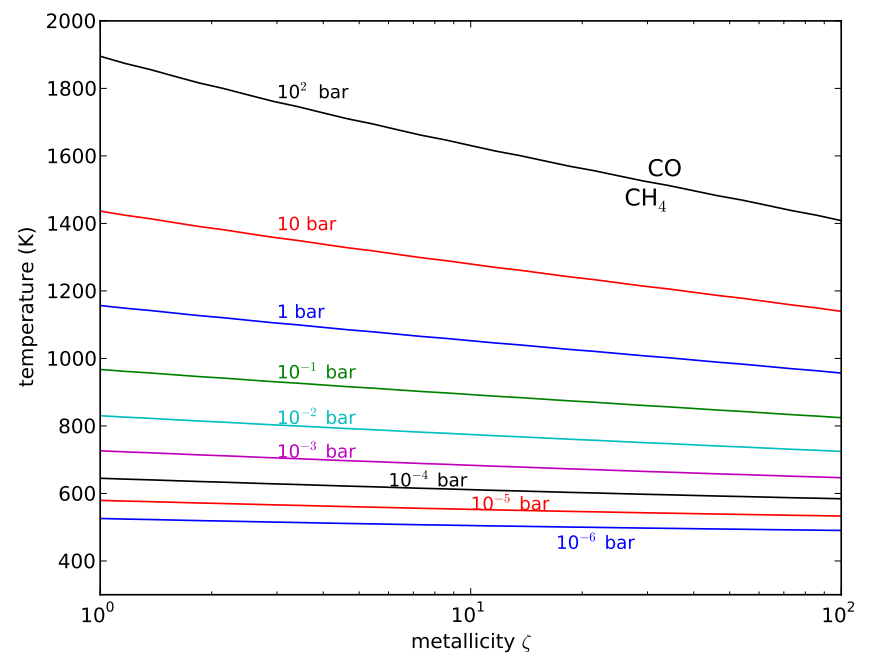

Fig. 1. Transition temperature for the C-bearing species (e.g. $\mathrm{CO} / \mathrm{CH}_{4}$ ) at the thermochemical equilibrium depending on metallicity at different pressures. Above the curve, $\mathrm{CO}$ is the dominant $\mathrm{C}$-bearing species, while $\mathrm{CH}_{4}$ dominates below. The temperature of the transition decreases when metallicity increases - an effect that is more important for high pressures.

and significantly cooler than a solar-type star, so that the planet is less severely heated (even if the orbital distances in the range 0.01-0.04 AU are as small as for hot Jupiters). This results in planetary effective temperatures below $1000 \mathrm{~K}$. Interestingly, it is around this temperature that gaseous mixtures with solar elemental abundances show a sharp transition concerning the major carbon reservoir, $\mathrm{CO}$ and $\mathrm{CH}_{4}$ as being the dominant carbon-containing species above and below $1000 \mathrm{~K}$, respectively (Fig. 1), under thermochemical equilibrium and at pressures around 1 bar. In this regard it is interesting to note that transit spectra of GJ 436b indicates that its atmosphere is poor in methane (Stevenson et al. 2010; Madhusudhan \& Seager 2011; Knutson et al. 2011), yet this species is predicted to be the major carbon reservoir at thermochemical equilibrium. Such interpretation has been, however, disputed by Beaulieu et al. (2011) based on a different analysis of transmission spectra. A detailed chemical model by Line et al. (2011), which considered thermochemical kinetics, vertical mixing, and photochemistry, concluded that $\mathrm{CH}_{4}$ should be the major carbon-bearing molecule in GJ 436b's atmosphere under most plausible conditions.

A second important difference with respect to hot Jupiters is that the lower mass of (sub-)Neptune planets allows us to expect an elemental atmospheric composition that is significantly enriched in heavy elements with respect to the solar composition because of their lower efficiency to retain light elements (Elkins-Tanton \& Seager 2008). In the case of GJ 1214b, its flat transmission spectrum indicates that the planet atmosphere is either hydrogen dominated but contains clouds or hazes, or has a high mean molecular weight, as exemplified in an $\mathrm{H}_{2} \mathrm{O}$-rich atmosphere (Bean et al. 2010, 2011; Désert et al. 2011; Croll et al. 2011; Crossfield et al. 2011; de Mooij et al. 2012; Berta et al. 2012). The possibility of a hydrogen-dominated atmosphere for GJ 1214b has been explored through chemical modelling by Miller-Ricci Kempton et al. (2012), who found that methane would be the major carbon reservoir. This is similar to the findings of Line et al. (2011)'s model on the atmosphere of GJ 436b. They found also that photolysis of $\mathrm{CH}_{4}$, which could lead to the formation of hazes, would take place at heights substantially higher than required by the observations. These previous photochemical studies dedicated to (sub-)Neptunes explored high metallicities up to $50 \times$ solar metallicity. In the solar system, Neptune and Uranus atmospheres indeed have carbon abundances about 50 times higher than in the Sun (oxygen being trapped in the deep and hot layers of the atmospheres, which cannot be probed yet by observations). This carbon abundance is significantly higher than that of the atmosphere of Jupiter and Saturn, which is about 3 times solar (Hersant et al. 2004). The bulk metallicity of icy giants is, however, much higher than that of their atmosphere, as they consist a large fraction of rocks and ice (e.g., Pollack et al. 1996; Alibert et al. 2005). In a warm Neptune that would have the same bulk composition as Neptune or Uranus, a larger fraction of the ices would be in the form of gases in the atmosphere, which may no longer be dominated by $\mathrm{H}_{2}$ and $\mathrm{He}$. Although the mass and radius of the planet, which derived from radial velocities and transit measurements, can be used to constrain the bulk metallicity, they do not provide a constraint on the metallicity of the envelope. This was shown by Baraffe et al. (2008) who modelled the evolution of Jupiter- and Neptune-mass planets with all the heavy elements located in the core or distributed uniformly in the whole planet. They also used different approximations to model the equation of state of the enriched envelope. Their conclusion is that the uncertainty related with the equation of state exceeds this difference, although planets with a uniform enrichment tend to have a smaller radius after about 1 Gyr compared with those with a core. Therefore, the mass and radius of a planet may show that a large fraction of the planet mass consists in $\mathrm{H}_{2}-\mathrm{He}$ but does not tell whether the envelope and the atmosphere are dominated by these compounds. The atmospheric abundance of $\mathrm{H}_{2} \mathrm{O}$, for instance, could reach or exceed that of $\mathrm{H}_{2}$, giving the atmosphere a high mean molecular weight and small scale height. In this study, we considered an enrichment in heavy elements between 1 and 100 times solar. Using solar abundances from Asplund et al. (2009) and assuming that all the oxygen is in the form of $\mathrm{H}_{2} \mathrm{O}$, this enrichment correspond to a mean molecular mass between 2.3 and $4.1 \mathrm{~g} / \mathrm{mole}$. Enrichment in heavy elements in the range $50-100$ is extremely interesting because it corresponds to a change of the carbon reservoir (either $\mathrm{CH}_{4}$ or $\mathrm{CO}$ ) for pressures within 1 and 100 bar and temperatures within 1000 and 2000 K (see Fig. 1). The deep atmospheric layers where such conditions are found can contaminate most of the atmosphere due to the chemical quenching associated with vertical mixing (e.g. Prinn \& Barshay 1977; Lewis \& Fegley Jr 1984; Visscher \& Moses 2011; Moses et al. 2011; Venot et al. 2012).

In this work, we address the effects of the heavy elements enrichment in the transiting warm Neptune GJ 3470b discovered by Bonfils et al. (2012). This planet is a promising candidate for follow-up characterisation of its atmosphere and for a better understanding of the atmospheric chemistry of (sub-)Neptunes. The planet GJ $3470 \mathrm{~b}$ has a mass of $14 M_{\oplus}$ in between those of GJ 436b (23 $M_{\oplus}$; Southworth 2010) and GJ 1214b (6 $M_{\oplus}$; Harpsøe et al. 2013). Its radius of $4.2 R_{\oplus}$ implies a high amount of hydrogen in the envelope. Indeed, a planet with the same mass but made only of water would have half this radius. Some spectroscopic observations during primary transit have already been held during the past few months (Demory et al. 2013; Fukui et al. 2013; Crossfield et al. 2013; Nascimbeni et al. 2013), leading sometimes to different and contradictory interpretations: hazy, cloud-free, metal-rich, low mean molecular weight, etc. Thus, more precise observations are needed to characterise its atmospheric structure and composition. While we wait for future observations, we study the composition of the atmosphere of GJ $3470 \mathrm{~b}$ with a model that includes thermochemical and 
Table 1. GJ 3470b's model parameters.

\begin{tabular}{lr}
\hline \hline Parameter & Value $^{a}$ \\
\hline Stellar radius & $0.503 R_{\odot}$ \\
Stellar effective temperature & $3600 \mathrm{~K}$ \\
Planetary radius & $4.2 R_{\oplus}$ \\
Planetary mass & $14.0 M_{\oplus}$ \\
Planet-star distance & $0.0348 \mathrm{AU}$ \\
\hline
\end{tabular}

Notes. ${ }^{(a)}$ Bonfils et al. (2012).

photochemical kinetics and vertical mixing. We explore the influence of the thermal profile, the vertical mixing efficiency, the poorly constrained UV irradiation, and the metallicity on the chemical composition. We compute the resulting transmission and emission spectra that we compare to the observations available so far. While this work was being finalised, a similar study has been published on GJ 436b by Moses et al. (2013). We do not compare our results to theirs in details, but they globally agree.

\section{Model}

We aim at studying the atmospheric chemical composition in the dayside of GJ 3470b in the vertical direction. We have adopted the planetary and stellar parameters derived by Bonfils et al. (2012), which are given in Table 1 . Note that the planetary parameters of GJ 3470 b have been recently refined by Demory et al. (2013) and Fukui et al. (2013), leading to a larger radius and consequently to a smaller density than what was predicted first by Bonfils et al. (2012). These new observations imply that GJ 3470b has a low density $\left(\rho_{p}<1 \mathrm{~g} \mathrm{~cm}^{-3}\right)$ compared to Uranus and Neptune. The atmosphere model relies on some key input information, such as the elemental composition, the vertical profile of temperature, the eddy diffusion coefficient, and the stellar ultraviolet (UV) flux, which are badly constrained. To explore the sensitivity of the atmospheric chemical composition to these uncertain parameters to some extent, we have varied them around some standard choices. Hereafter, we describe our choice of standard parameters and the range over which they have been varied.

\subsection{Stellar spectrum}

The radiation spectrum of the host star affects the planetary atmosphere in two major ways. On the one hand, the visibleinfrared part of the incoming stellar radiation controls the atmospheric thermal structure of the planet, and on the other, the $\mathrm{UV}$ radiation determines the photodissociation rates. Longward of $240 \mathrm{~nm}$, we adopt a Phoenix NextGen synthetic spectrum (Hauschildt et al. 1999) for a star with $T_{\mathrm{eff}}=3600 \mathrm{~K}, g=$ $10^{4.5} \mathrm{~cm} \mathrm{~s}^{-2}$, and solar metallicity. The flux of $\mathrm{UV}$ radiation emitted by $M$ dwarf stars may vary by orders of magnitude depending of the degree of chromospheric activity of the star (France et al. 2013). Unfortunately, the UV spectrum of GJ 3470 has not been observed to our knowledge, and we have therefore adopted both the observed spectrum of the active M3.5V star GJ 644 (Segura et al. 2005) in the 115-240 nm wavelength range and the mean of the Sun spectra at maximum and minimum activity (Gueymard 2004) shortward of $115 \mathrm{~nm}$. The final composite spectrum (shown in Fig. 2) is adopted as the standard stellar spectrum. When exploring the space of parameters, we allow for a variation in the UV flux between 0.1 and 10 times the standard spectrum, due to the large uncertainties of the stellar flux.

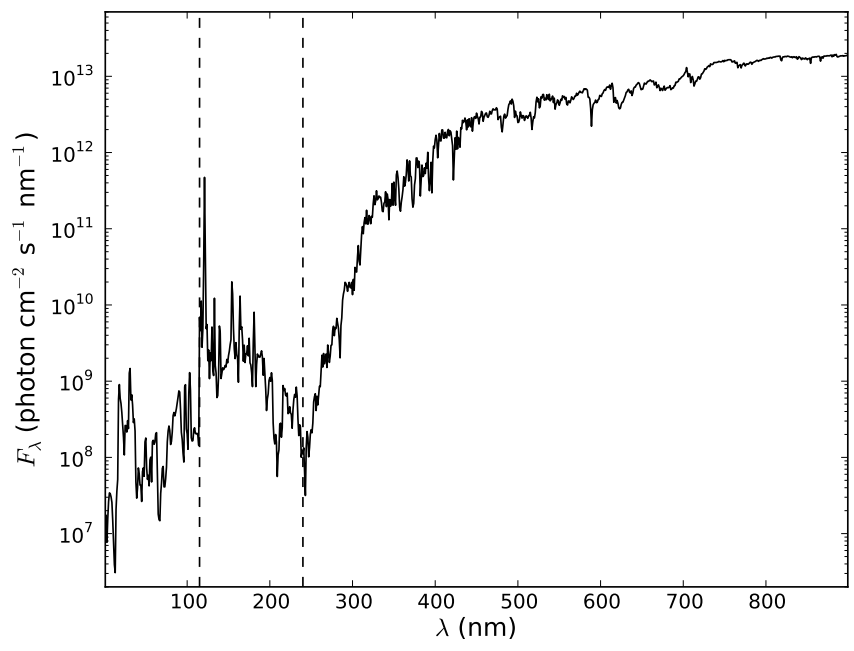

Fig. 2. Stellar spectrum adopted for GJ 3470, where the flux $F_{\lambda}$ is normalised to an orbital distance of $1 \mathrm{AU}$. Vertical dashed lines at 115 and $240 \mathrm{~nm}$ indicate the positions of junction between spectra from different sources (see text).

\subsection{Atmospheric metallicity}

To understand the history of GJ 3470b, it would be important to constrain its atmospheric metallicity, which is currently very uncertain. As it has been explained in the Introduction, the atmosphere of this planet can be enriched. The host star of GJ 3470b has a metallicity slightly above the solar value, $[\mathrm{Fe} / \mathrm{H}]=+0.2$, according to Demory et al. (2013). In our standard model, we consider that the abundances of heavy elements (other than $\mathrm{H}$ and $\mathrm{He}$ ) are enriched in GJ 3470b's atmosphere by a factor of $\zeta=10$ with respect to the solar values compiled by Asplund et al. (2009). However, given the large uncertainties in the elemental composition, we choose two extreme cases with $\zeta=1$ (solar metallicity) and $\zeta=100$ (high metallicity) when exploring the effect of metallicity on the atmospheric chemical composition. In this study, we do not change the $\mathrm{C} / \mathrm{N} / \mathrm{O}$ relative abundance ratios compared to their solar values.

\subsection{Thermal profile}

The vertical profile of temperature in GJ 3470b's atmosphere is computed with the radiative-convective model described by Iro et al. (2005) with the update of Agúndez et al. (2012). We adopt the planetary and stellar parameters given in Table 1, as well as the input information corresponding to our standard model. The mixing ratios of the main species that provide opacity are estimated through thermochemical equilibrium, which is expected to be a good approximation as long as the abundances of $\mathrm{CO}$ and $\mathrm{H}_{2} \mathrm{O}$ (the main species that affect the thermal structure) are close to the chemical equilibrium values. As seen in Sect. 3.1, this is likely to be the case for $\mathrm{H}_{2} \mathrm{O}$ although not for $\mathrm{CO}$ throughout a good part of the atmosphere, which may add an uncertainty to the calculated thermal profile. In the deep atmosphere, the temperature is regulated by convective, rather than radiative, processes, and the internal flux of the planet becomes the most relevant parameter. The internal flux of the planet is highly uncertain since it depends on the age of the planet and on processes of dissipation of energy, which may be triggered by tidal effects (Agúndez et al. 2014). We have adopted an internal flux, which corresponds to an internal temperature of $100 \mathrm{~K}$, a value commonly used in previous studies in the absence of relevant constraints. The temperature is calculated vertically as a function of 


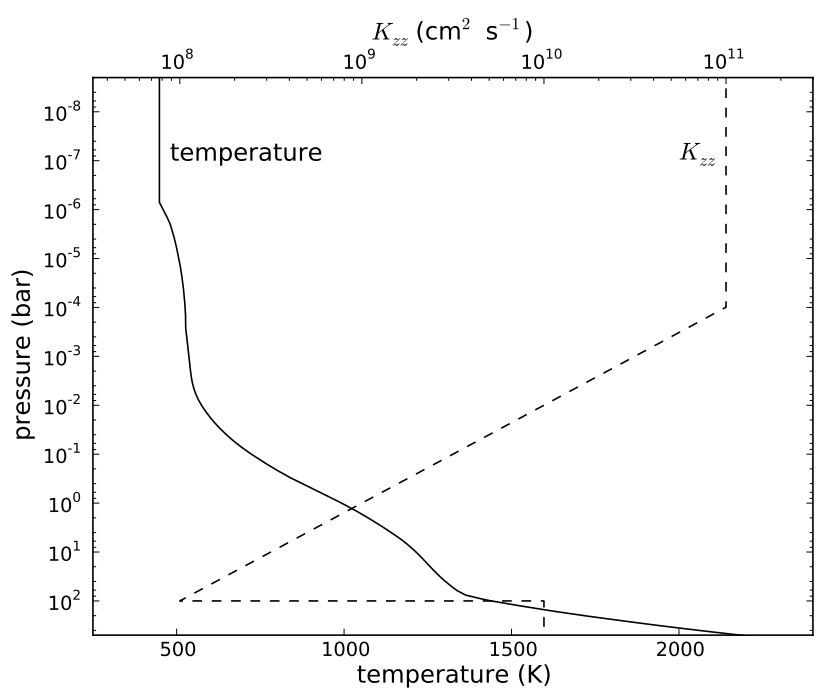

Fig. 3. Standard vertical profile of temperature (solid line referred to the lower abscissa axis) and of eddy diffusion coefficient (dashed line referred to the upper abscissa axis) adopted for the atmosphere of GJ 3470b.

pressure between 1000 and $10^{-6}$ bar, and above this latter pressure level, an isothermal atmosphere is assumed. The calculated vertical profile of temperature, which is adopted as the standard one, is shown in Fig. 3. Given the various uncertainties that affect the calculated temperature profile, we explore this in our space of parameters by choosing two bounding cases in which a value of $100 \mathrm{~K}$ is added and subtracted to the standard temperature profile.

\subsection{Vertical mixing}

Another important parameter for the chemical model is the vertical profile of the eddy diffusion coefficient, which determines the efficiency of the vertical mixing as a function of pressure. In the case of exoplanet atmospheres, constraints on this parameter come solely from global circulation models (GCMs). For the atmosphere of GJ 3470b, we adopt a parametric profile for the eddy diffusion coefficient with a high value of $K_{z z}=$ $10^{10} \mathrm{~cm}^{2} \mathrm{~s}^{-1}$ in the convective region of the atmosphere (which is approximately located below the 100 bar pressure level) and values inferred from the GCM of GJ 436b developed by Lewis et al. (2010). By multiplying a mean vertical wind speed by the local scale height, these authors estimated $K_{z z}$ values of $10^{8} \mathrm{~cm}^{2} \mathrm{~s}^{-1}$ at 100 bar and $10^{11} \mathrm{~cm}^{2} \mathrm{~s}^{-1}$ at $0.1 \mathrm{mbar}$. We have therefore adopted these values and assumed a linear behaviour in the logarithm of $K_{z z}$ with respect to the logarithm of pressure in the $10^{-4}-100$ bar regime, and a constant value for $K_{z z}$ at higher atmospheric layers. The resulting vertical profile, which we adopt as the standard one, is shown in Fig. 3 as referred to the upper abscissa axis. However, because the GCM of Lewis et al. (2010) is constructed for GJ 436b and not for GJ 3470b, and also because the method used to estimate the eddy diffusion coefficient is highly uncertain (e.g. Parmentier et al. 2013), we have explored the sensitivity of the chemical abundances to the eddy diffusion coefficient. We have considered two limiting cases in which $K_{z z}$ is divided and multiplied by a factor of ten with respect to the standard profile above the convective region.

\subsection{Kinetics}

Once the physical parameters and elemental composition are established, the atmospheric chemical composition is computed by solving the equation of continuity in the vertical direction for 105 species composed of $\mathrm{H}, \mathrm{He}, \mathrm{C}, \mathrm{N}$, and $\mathrm{O}$. The reaction network and photodissociation cross sections used are described in Venot et al. (2012). This chemical network, which includes $\sim 1000$ reversible reactions (so a total of $\sim 2000$ reactions), has been developed from applied combustion models and has been validated over a large of temperature (from 300 to $2500 \mathrm{~K}$ ) and pressure (from a few mbar to some hundred of bar). It is able to reproduce the kinetic evolution of species with up to two carbon atoms. Thus, our chemical network is valid to study the chemical composition of the atmosphere of GJ 3470b.

We can compare our results with previous results obtained for other (sub-)Neptune atmospheres, as exemplified by Line et al. (2011; GJ 436b) and Miller-Ricci Kempton et al. (2012; GJ 1214b). Both studies use smaller chemical networks compared to ours $(\sim 700$ reactions with 51 and 61 species, respectively) and reverse all reaction rates using the principle of microscopic reversibility (Visscher \& Moses 2011; Venot et al. 2012). However, contrary to our network, none of them have been validated as a whole through experiments. Line et al. (2011) use the chemical network conceived for Jovian planets (Liang et al. 2003, 2004, and reference therein), which has been updated for high temperature (Line et al. 2010) and enhanced with nitrogen reactions and a small set of $\mathrm{H}_{2} \mathrm{~S}$ reactions. Miller-Ricci Kempton et al. (2012) use the chemical network of Zahnle et al. (2009b), which is also originally made for Jovian planets (Zahnle et al. 1995) and upgraded for high temperature atmospheres with an arbitrary selection of new reaction rates from available data (Zahnle et al. 2009a). As it has been shown in Venot et al. (2012), different chemical schemes can lead to different quenching levels and thus to differences in computed atmospheric composition. Thus, some differences found between this study and those from Line et al. (2011) and Miller-Ricci Kempton et al. (2012) may be due to the use of different chemical schemes.

\section{Results and discussion}

Our standard set of parameters to build up the chemical model of GJ 3470b's atmosphere consists of an elemental composition given by $\zeta=10$, the vertical profiles of temperature, eddy diffusion coefficient shown in Fig. 3, and the stellar UV spectrum shown in Fig. 2. Apart from this standard model, we have constructed a grid of 16 models in which we have explored the sensitivity of the chemical composition to the metallicity, temperature, eddy diffusion coefficient, and stellar UV flux, according to the choices detailed in Table 2 . For all of the seventeen models, the initial conditions are the thermochemical equilibrium. At both upper and lower boundaries, we impose a zero flux for each species. The steady-state is reached after an integration time of $t=10^{8} \mathrm{~s}\left(K_{z z}^{\times 10}\right)$ or $t=10^{9} \mathrm{~s}\left(K_{z z}^{\div 10}\right)$.

\subsection{Standard model}

In this section, we present the results of our standard model and compare them with previous publications dealing with (sub-)Neptunes: Line et al. (2011) on GJ 436b and Miller-Ricci Kempton et al. (2012) on GJ 1214b. Because these models do not use the same thermal profiles as noted here, the same eddy diffusion profiles, and elemental abundances, it is difficult to quantitatively compare our results. Nevertheless, 
Table 2. Parameter space of the model explored.

\begin{tabular}{lll}
\hline \hline Parameter & Range of values & Symbol \\
\hline Metallicity & Solar $(\zeta=1)$ & $\zeta_{1}$ \\
& High $(\zeta=100)$ & $\zeta_{100}$ \\
\hline Temperature & Warm atmosphere $(+100 \mathrm{~K})$ & $T_{+100}$ \\
& Cool atmosphere $(-100 \mathrm{~K})$ & $T_{-100}$ \\
\hline Eddy diffusion coefficient & High $\left(K_{z z} \times 10\right)$ & $K_{z z}^{\times 10}$ \\
& Low $\left(K_{z z} \div 10\right)$ & $K_{z z}^{\div 10}$ \\
\hline Stellar UV flux & High irradiation $\left(F_{\lambda} \times 10\right)$ & $F_{\lambda}^{\times 10}$ \\
& Low irradiation $\left(F_{\lambda} \div 10\right)$ & $F_{\dot{\lambda}}^{\div 10}$ \\
\hline
\end{tabular}

Notes. All the parameters are changed with respect to the standard values showed in Figs. 2 and 3. The standard metallicity is $10 \times$ solar $(\zeta=10)$.

different cases have been studied in these publications, so we can qualitatively compare the results that we obtained.

\subsubsection{Chemical composition}

Figure 4 shows the atmospheric composition of GJ 3470b at the chemical equilibrium and at the steady-state, computed with the model taking into account thermochemical kinetics, vertical mixing, and photochemistry. The abundances of all species remain at chemical equilibrium for pressures higher than about 40 bar, while we can see the effect of vertical mixing at lower pressures. Around 40 bar the abundances of $\mathrm{HCN}$ and $\mathrm{NH}_{3}$ depart from chemical equilibrium, and at lower pressure, around $2 \mathrm{bar}$, the abundances of $\mathrm{CO}_{2}, \mathrm{CO}, \mathrm{CH}_{4}$, and $\mathrm{H}_{2} \mathrm{O}$ get quenched. That is, they are frozen at the chemical equilibrium value of the quench level. This quenching effect makes $\mathrm{CH}_{4}, \mathrm{H}_{2} \mathrm{O}$, and $\mathrm{N}_{2}$ slightly less abundant than what thermochemical equilibrium would predict, so that $\mathrm{CO}, \mathrm{NH}_{3}, \mathrm{CO}_{2}$, and $\mathrm{HCN}$ can be more abundant than the equilibrium prediction. In the upper atmosphere (above the $10^{-6}$ bar level), we see the effect of photodissociations: some species (for example $\mathrm{H}_{2} \mathrm{O}$ and $\mathrm{CH}_{4}$ ) are destroyed by photolysis, whereas others (as $\mathrm{CO}_{2}$ and $\left.\mathrm{CO}\right)$ see their abundance increased. Globally, the most abundant species of the atmosphere of GJ 3470b (after $\mathrm{H}_{2}$ and $\mathrm{He}$ ) between $10^{2}$ and $10^{-6}$ bar are $\mathrm{H}_{2} \mathrm{O}, \mathrm{CH}_{4}$, and $\mathrm{CO}$ by decreasing order.

First, we compare our results with those of Line et al. (2011). We focus on the cases, where elemental abundances are solar and $50 \times$ solar. Our $T-P$ profile is not very different from theirs so we expect to have similar results. Even if our eddy diffusion coefficient is not identical, the abundances we find for all species are in between these two cases. In the region where vertical quenching dominates (in between the thermochemical equilibrium and photochemical regions), the behaviour of abundances is similar since the eddy diffusion coefficient adopted for the quenching level is not very different $\left(10^{8} \mathrm{~cm}^{2} \mathrm{~s}^{-1}\right.$ by Line et al. (2011) and less than $10^{9} \mathrm{~cm}^{2} \mathrm{~s}^{-1}$ in our case). However, our adopted $K_{z z}$ value in the upper layers is substantially higher than the value of $10^{8} \mathrm{~cm}^{2} \mathrm{~s}^{-1}$ adopted by Line et al. (2011), so that the region where photochemistry takes place in their models is shifted to lower heights.

Then, we compare our results with those obtained by Miller-Ricci Kempton et al. (2012) using $5 \times$ and $30 \times$ solar elemental abundances and an eddy diffusion coefficient of $K_{z z}=$ $10^{9} \mathrm{~cm}^{2} \mathrm{~s}^{-1}$. We expect our results to be in between these two results. That is what we find for most species, except

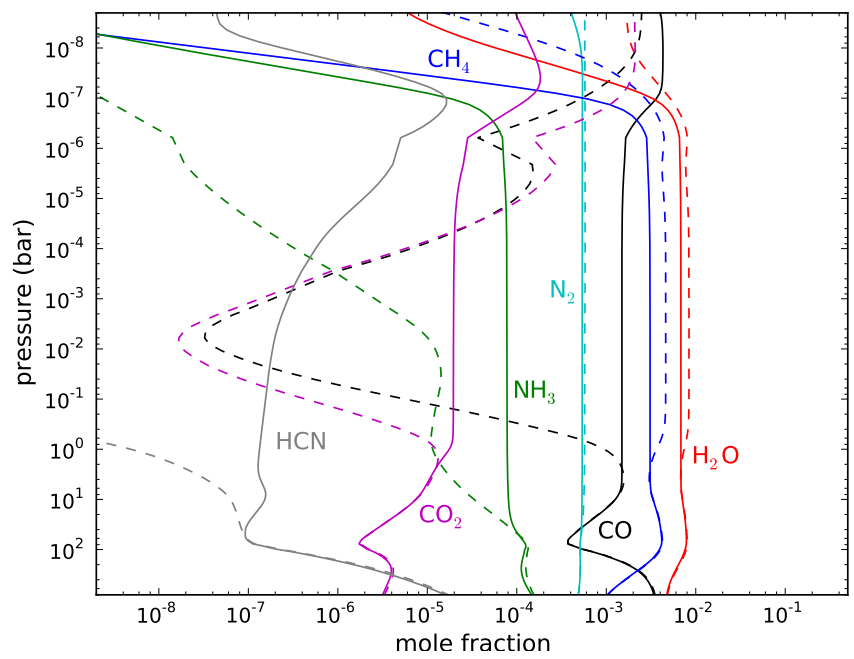

Fig. 4. Vertical distribution of molecular abundances in the standard model of GJ 3470b's atmosphere as computed through thermochemical equilibrium (dashed lines) and with the model that includes thermochemical kinetics, vertical mixing, and photochemistry (solid lines).

$\mathrm{CO}$ and $\mathrm{CO}_{2}$. For these two species at the steady-state, our model gives abundances about 100 times higher than in their case $\zeta=30$. This is because the abundances of these species depart from chemical equilibrium at a higher pressure in the study of Miller-Ricci Kempton et al. (2012) when compared to our results $\left(\sim 10^{2}\right.$ bar and $\sim 5$ bar, respectively). Indeed, Miller-Ricci Kempton et al. (2012) use a similar thermal profile, except for pressures higher than 1 bar. While the temperature increases with pressure in our $T-P$ profile, the temperature remains constant between 1 and 100 bar in their model. Consequently, the temperature in the deeper part of the atmosphere, where quenching happens, is colder than in our $T-P$ profile. This difference has consequences on the abundances of some species at the chemical equilibrium (For a given pressure level, $\mathrm{CO}$ and $\mathrm{CO}_{2}$ have equilibrium abundances smaller than in our model.) and also at the steady-state because quenching happens at different levels.

\subsection{2. $\mathrm{CH}_{4} / \mathrm{CO}$ abundance ratio}

The $\mathrm{CH}_{4} / \mathrm{CO}$ abundance ratio is an important parameter to discuss, since some observational and modelling studies seem to indicate a poor methane content in the atmosphere of warm (sub-)Neptunes, while thermochemical equilibrium predicts that $\mathrm{CH}_{4}$ should be the major carbon reservoir in such atmospheres (e.g Stevenson et al. 2010; Madhusudhan \& Seager 2011; Knutson et al. 2011 for GJ 436b and Miller-Ricci Kempton et al. 2012 for GJ 1214b). Of course, chemical equilibrium depends on the $T-P$ profile and the assumed elemental composition, but these findings have suggested the need to invoke non-equilibrium processes, such as mixing and photodissociations to help explain these non-expected chemical compositions. Nevertheless, even taking into account these non-equilibrium processes, 1D chemical models have not been able to find the set of parameters that may lead to a $\mathrm{CH}_{4} / \mathrm{CO}$ abundance ratio lower than 1 . In the case of the warm Neptune GJ 436b, observations of the dayside emission seem to indicate that this planet has an atmosphere dominated by $\mathrm{CO}$ and is poor in $\mathrm{CH}_{4}$ $\left(\mathrm{CH}_{4} / \mathrm{CO}\right.$ abundance ratio equals to $10^{-4}-10^{-3}$ for Stevenson et al. 2010 and Madhusudhan \& Seager 2011), although a different interpretation has been provided by Beaulieu et al. (2011), 
based on the re-analysis of the same secondary eclipse data and on primary transit observations. This indicates a high methane content in the atmosphere with eventually traces of $\mathrm{CO}$ or $\mathrm{CO}_{2}$. Whatever the right interpretation, the chemical modelling done by Line et al. (2011) shows that $\mathrm{CH}_{4}$ is more abundant than $\mathrm{CO}$ (the $\mathrm{CH}_{4} / \mathrm{CO}$ abundance ratio is $\sim 2 \times 10^{3}$ and $\sim 3$ between $10^{-3}$ and 1 bar for metallicities of $\zeta=1$ and 50, respectively). In the case of GJ 1214b, Miller-Ricci Kempton et al. (2012) find that $\mathrm{CH}_{4}$ should be the major carbon reservoir in the atmosphere with $\mathrm{CH}_{4} / \mathrm{CO}$ abundance ratios of $\sim 6 \times 10^{4}$ and $\sim 10^{3}$ between $10^{-2}$ and 1 bar for metallicities of $\zeta=5$ and 30 but also find that it is a no- $\mathrm{CH}_{4}$ model that best fits the observations of this planet.

With the standard value of the parameters of GJ 3470b, we find a $\mathrm{CH}_{4} / \mathrm{CO}$ abundance ratio of 2 at 1 bar, with $\mathrm{CH}_{4}$ being slightly more abundant than $\mathrm{CO}$. We then explore how the $\mathrm{CH}_{4} / \mathrm{CO}$ abundance ratio varies within the space of parameters.

\subsection{The parameter space of $\zeta, T, K_{\mathrm{zz}}$, and $F_{\lambda}$}

We study the different possible atmospheric compositions of GJ 3470 b by exploring the space of unknown parameters: metallicity $(\zeta)$, temperature $(T)$, eddy diffusion coefficient $\left(K_{z z}\right)$, and incident UV flux $\left(F_{\lambda}\right)$. The computed abundances of $\mathrm{CO}, \mathrm{CO}_{2}$, $\mathrm{CH}_{4}, \mathrm{NH}_{3}, \mathrm{H}_{2} \mathrm{O}$, and $\mathrm{HCN}$ are plotted in Fig. 5. We choose these species because they are the ones that most influence the planetary spectra.

\subsubsection{Effect of metallicity}

An increase in the metallicity obviously produces an abundance enhancement of all molecules containing heavy atoms (compare red and magenta line, or green and blue line, in Fig. 5). Apart from this, the reaction to a change in the metallicity can be different, depending of the molecule with the most sensitive one being carbon dioxide. When the metallicity changes from $\zeta=1$ to 100 , the abundance of $\mathrm{CO}_{2}$ increases by $4-6$ orders of magnitude, while that of CO increases by $2-4$ orders of magnitude. The rest of the molecules experience less dramatic variations. A large abundance of $\mathrm{CO}_{2}$ would probably be the best evidence of an enhanced metallicity in the planet's atmosphere, as already found by Zahnle et al. (2009b) in the case of hot Jupiters. Nitrogen species are also sensitive to metallicity. Both $\mathrm{N}_{2}$ and $\mathrm{HCN}$ increase their abundance by $\sim 2$ orders of magnitude when metallicity increases.

\subsubsection{Effect of temperature}

At the typical temperature range expected in the atmosphere of a warm Neptune, such as GJ 3470b, a variation in temperature of $200 \mathrm{~K}$ can produce important changes in the resulting chemical abundances. These abundance variations depend to a large extent on the adopted metallicity. If we focus on the most abundant molecules, which are shown in Fig. 5, there are two clear behaviours. On the one hand, we have $\mathrm{CO}, \mathrm{CO}_{2}$, and $\mathrm{HCN}$, which experience an abundance enhancement when the temperature is increased, especially at low metallicities $(\zeta=1)$. In this case, the abundances of these molecules vary by 1-2 orders of magnitude. We notice that the change of temperature has less effect at high metallicities $(\zeta=100)$. The molar fraction of $\mathrm{CO}$ and $\mathrm{HCN}$ vary only by a factor $\sim 2$, whereas the abundance of $\mathrm{CO}_{2}$ exhibits negligible change. On the other hand, $\mathrm{CH}_{4}, \mathrm{H}_{2} \mathrm{O}$, and $\mathrm{NH}_{3}$ respond to an increase in temperature in the opposite direction, which decreases their abundances. In this case, the effect is more apparent at high metallicities with abundance variations up to one order of magnitude.

The chemical composition in the atmosphere of warm (sub-)Neptunes can be sensitive to the temperature, especially if the temperature is around $1000 \mathrm{~K}$ in the quench region that is usually located in the $0.1-10$ bar pressure range. At these temperatures and pressures, there are important transitions concerning $\mathrm{CO}$ and $\mathrm{CH}_{4}$. Uncertainties in the thermal profile are therefore a major source of error in some of the calculated abundance ratios.

\subsubsection{Effect of vertical mixing}

When there is stronger vertical mixing, quenching occurs deeper, and the upper atmosphere will be more contaminated by the chemical composition of the deep atmosphere. This has a crucial importance for interpreting the observations and thus the composition of such atmospheres. Globally, a higher eddy diffusion coefficient results in a stronger vertical mixing, so the abundance profiles are more flat in the vertical direction. For $\mathrm{CO}, \mathrm{CO}_{2}$, and $\mathrm{HCN}$, a high $K_{z z}$ leads to smaller abundances than with a low $K_{z z}$ at pressures above the quenching level, whereas a high $K_{z z}$ creates globally higher abundances for all the other species.

\subsubsection{Effect of stellar UV flux}

For some species ( $\mathrm{HCN}, \mathrm{CO}_{2}$ and $\mathrm{CO}$ ), the effect of photochemistry propagates deep in the atmosphere down to few bars, thanks to vertical mixing. For these species (if $\mathrm{CH}_{4}$ is reservoir of carbon), the effect of photochemistry is to enhance their abundance, especially at low metallicity. A more intense UV flux results in an increase in their abundance. For the other species represented in Fig. 5, the effect of the UV flux remains only at low pressures $\left(<10^{-3}\right.$ bar $)$. These species are destroyed by photolysis, so a higher photochemistry shifts photodestruction of molecules to lower heights.

\subsection{Combined effect of the parameters on the $\mathrm{CH}_{4} / \mathrm{CO}$ abundance ratio}

The main finding of this paper is that there exists a combined effect of the temperature, the vertical mixing, and the metallicity that can explain the $\mathrm{CH}_{4} / \mathrm{CO}$ abundance ratio that is lower than unity, which has been found by observations in some atmospheres. To clearly see the dependence of the $\mathrm{CH}_{4} / \mathrm{CO}$ ratio with the parameters of our study, we plot the two more extreme cases, $K_{z z}^{\times 10} F_{\lambda}^{\div 10}$ and $K_{z z}^{\div 10} F_{\lambda}^{\times 10}$ (Fig. 6) for each $\zeta-T$ choice. The two other cases are in-between.

In the cases of low and standard metallicity, the $\mathrm{CH}_{4} / \mathrm{CO}$ abundance ratio is always above 1 (for pressures higher than $10^{-2}$ mbar) for whatever the choice of the parameters $T, K_{z z}$ and $F_{\lambda}$ (4 top panels in Fig. 6). The maximum value reached is $\sim 200$ between $10^{-3}$ and 1 bar (with the case $\zeta_{100} T_{-100}$ ). When the metallicity is increased up to $\zeta=100$, we find that the $\mathrm{CH}_{4} / \mathrm{CO}$ abundance ratio may become lower than unity if a warm temperature profile is adopted. In the cases of $\zeta_{100} T_{+100}$ (magenta curves in Fig. 5), for a given $K_{z z}$ and $F_{\lambda}, \mathrm{CO}$ is clearly more abundant than $\mathrm{CH}_{4}$ (In the area $10^{-3}-1$ bar, $\mathrm{CH}_{4} / \mathrm{CO}$ ranges between 0.04 and 0.06.). We can see that this result is determined by the thermodynamic equilibrium in Fig. 6 (bottom panels): Because the temperature is high in the deep atmosphere, $\mathrm{CO}$ is thermochemically favoured over $\mathrm{CH}_{4}$. Vertical mixing then makes the abundance of $\mathrm{CO}$ and $\mathrm{CH}_{4}$ to quench in the vertical direction, so that $\mathrm{CO}$ remains more abundant than methane in all 

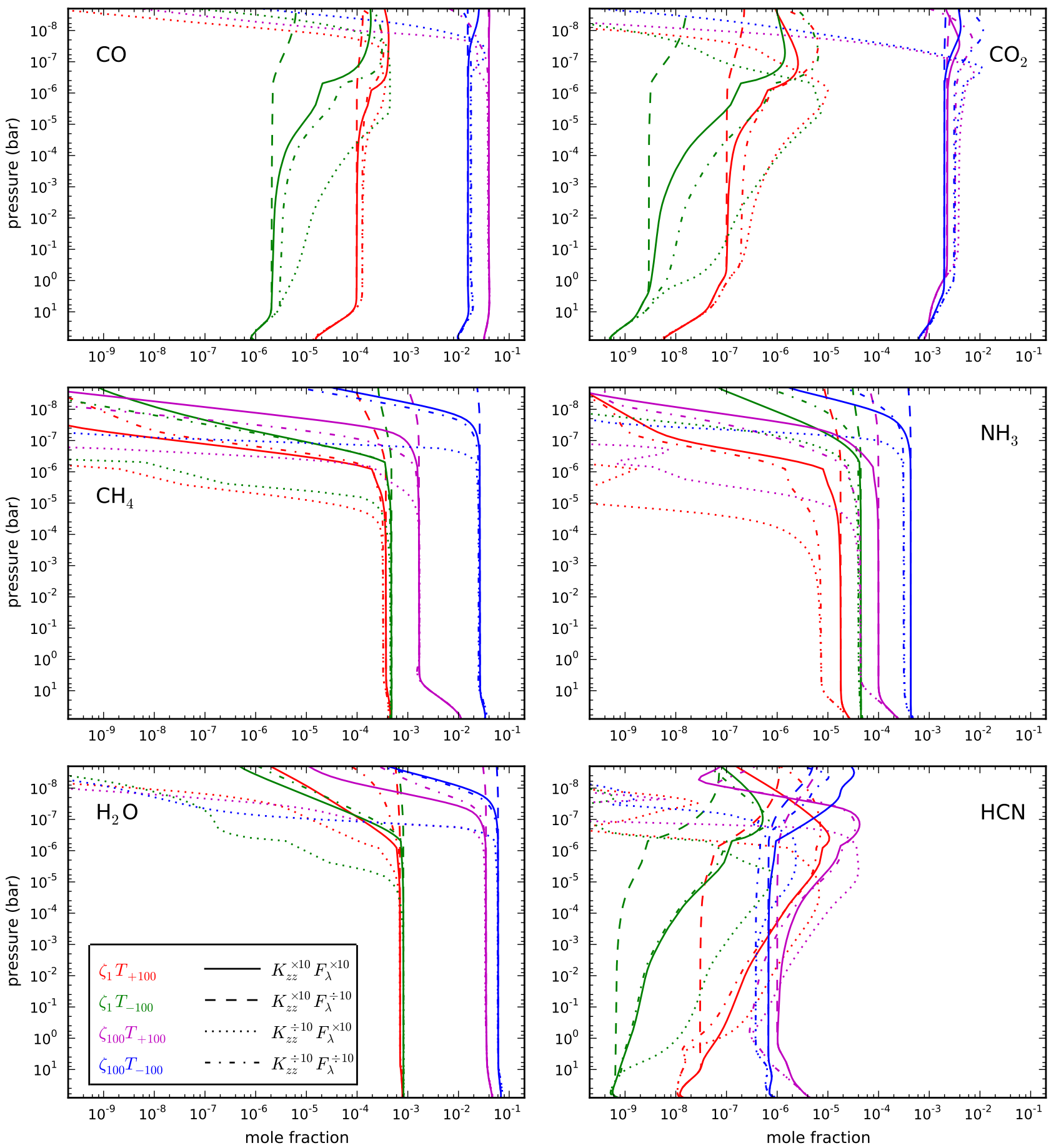

Fig. 5. Vertical distribution of the abundances of selected molecules as calculated through each of the 16 models in which the space of metallicity, temperature, eddy diffusion coefficient, and stellar UV flux are explored. Each colour corresponds to a set of metallicity and temperature values and each line style to a set of eddy diffusion coefficients and stellar UV fluxes (see legend in the $\mathrm{H}_{2} \mathrm{O}$ panel and meaning of each symbol in Table 2).

the upper atmosphere. This occurs despite thermochemical equilibrium predicts an inversion of C-bearing species between $\sim 0.7$ and 100 mbar. Nevertheless, we find a $\mathrm{CH}_{4} / \mathrm{CO}$ ratio higher than 1 for the cases $\zeta_{100} T_{-100}$ (blue curves in Fig. 5). This indicates that not only a high metallicity is necessary to a $\mathrm{CH}_{4} / \mathrm{CO}$ ratio under unity but also a sufficiently high internal temperature.

These results show that it is possible for GJ $3470 \mathrm{~b}$ but also for the observed GJ $436 \mathrm{~b}$ to have a $\mathrm{CH}_{4} / \mathrm{CO}$ ratio under unity. This is true to a lesser extent for GJ $1214 \mathrm{~b}$ because of its lower temperature. A very high metallicity, when compared to the Sun, combined with a high temperature may be the key to explain the observations of warm exoplanets, such as GJ 3470b, which indicate that $\mathrm{CO}$ is more abundant than methane. Moreover, planets with a bulk composition similar to Neptune or Uranus are expecting to have such enrichment. The study also shows that getting back to the elementary abundances from observations is very difficult and requires knowing the temperature profile, the metallicity, and the vertical mixing. The solution might be to use 
A\&A 562, A51 (2014)
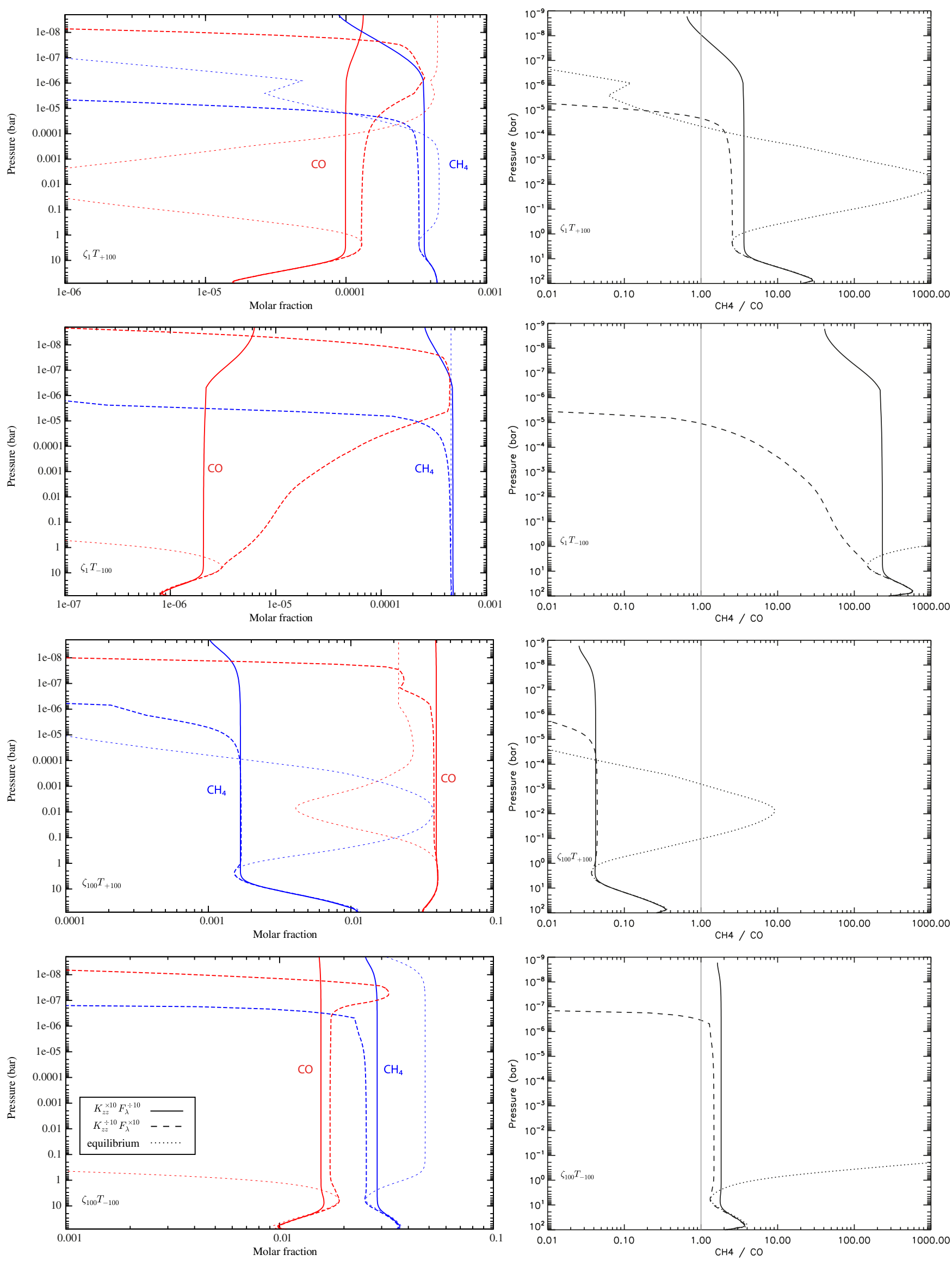

Fig. 6. Vertical abundances of $\mathrm{CO}$ and $\mathrm{CH}_{4}$ in eight selected models (left) and the corresponding value of the $\mathrm{CH}_{4} / \mathrm{CO}_{\text {ratio }}($ right). Each line style corresponds to a set of eddy diffusion coefficients and stellar UV fluxes (see legend in the left bottom panel and meaning of each symbol in Table 2). The $\mathrm{CH}_{4} / \mathrm{CO}=1$ line is represented with a grey line. 


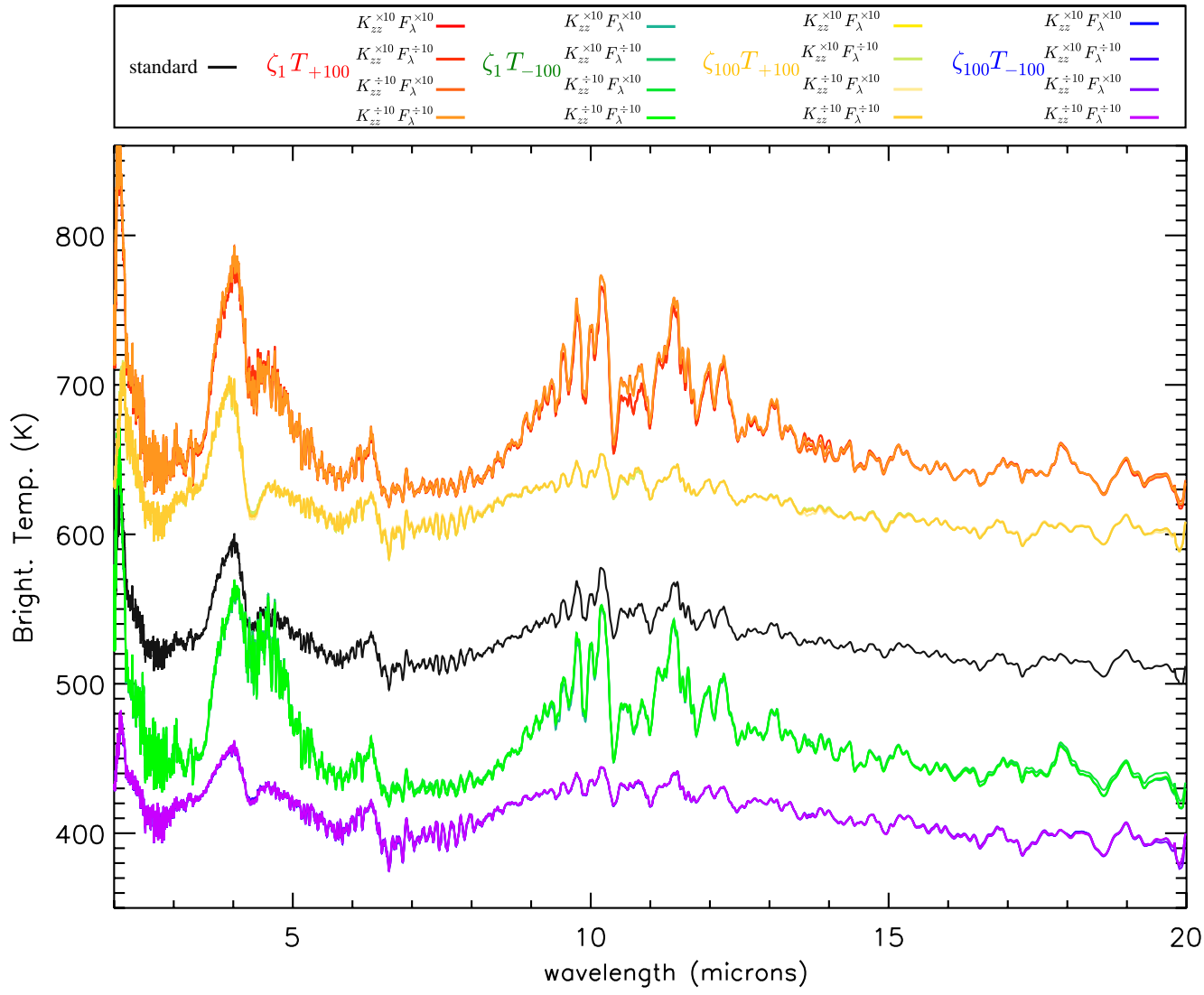

Fig. 7. Synthetic emission spectra of GJ $3470 \mathrm{~b}$ corresponds to the grid of 16 models and the standard model. Each colour corresponds to a set of metallicity and thermal profile. A colour gradient is then used to differentiate the eddy diffusion coefficients and stellar UV fluxes (see legend in the top panel and meaning of each symbol in Table 2). The standard values are shown in Figs. 2 and 3. The standard metallicity is $10 \times$ so$\operatorname{lar}(\zeta=10)$. a self-consistent model, which takes into account and calculates simultaneously all these parameters.

\subsection{Synthetic spectra}

To determine if future observations of GJ $3470 \mathrm{~b}$ could be used to constrain the values of the parameters that we varied in the previous section, we compute synthetic spectra for our 17 models (Fig. 7). Emission spectra are calculated using the line-by-line radiative transfer codes described in Tinetti et al. (2005, 2006). Transmission spectra are calculated using the line-by-line radiative transfer codes described in Hollis et al. (2013). For both type of spectra, we used line lists from HITRAN (Rothman et al. 2009, 2010), except for $\mathrm{CO}, \mathrm{CO}_{2}$, and $\mathrm{CH}_{4}$ for which we used HITEMP (Rothman et al. 2010). For $\mathrm{H}_{2} \mathrm{O}$, we used the BT2 list (Barber et al. 2006). We use the same NextGen stellar model as in Sect. 2.1.

We end up with five groups of spectra influenced by the thermal profile and the metallicity. The vertical mixing, as well as the UV flux used in the different models, have very little effect on spectra, which are dominated by the temperature and the metallicity of the atmosphere. For both primary and secondary spectra, we notice that the reddish and greenish spectra exhibit broader variations than the two others, because they correspond to the low metallicity cases, so to atmospheres with smaller optical depth. This result has also been found by Agúndez et al. (2014) and Moses et al. (2013) for GJ 436b.

Thereby, we see five levels of brightness temperature for emission spectra. The highest temperature corresponds to the case of $\zeta_{1} T_{+100}$ (reddish curves), and the lowest to the case of $\zeta_{100} T_{-100}$ (blueish curves). The standard model is logically in between the four other groups of spectra. With an identical thermal profile, the enhancement of metallicity (by a factor 100) leads to a lowering of the brightness temperature by $\sim 50 \mathrm{~K}$. The optical depth of the atmosphere increases together with the metallicity. Thus, the signal received during the secondary transit comes from a higher level in the atmosphere, so it corresponds to lower temperatures, providing such differences of brightness temperature. Of course, in the case of temperature inversion, one could have the opposite effect, and see higher brightness temperatures with higher metallicities. Constraining the metallicity from the brightness temperature is difficult because of the strong dependency of the latter on the temperature; therefore, there is a degeneracy. Apart from the level of brightness temperature, the spectra are globally similar and exhibit the same features. Nevertheless, we can notice slight differences between the $\zeta_{1}$ and $\zeta_{100}$ spectra at two locations. First, the absorption by $\mathrm{CO}_{2}$ and $\mathrm{CO}$ at around $4.5 \mu \mathrm{m}$ are more defined in the $\zeta_{100}$ cases compared to the very close peaks that are characteristic of water absorption that we see around $4.5 \mu \mathrm{m}$ in the $\zeta_{1}$ cases. Around $10 \mu \mathrm{m}$, the high peaks due to $\mathrm{NH}_{3}$ and $\mathrm{H}_{2} \mathrm{O}$ are very strong features in the $\zeta_{1}$ spectra but are attenuated on the $\zeta_{100}$ spectra. This is due to the high abundance of $\mathrm{CO}_{2}$, which absorbs a lot from $9 \mu \mathrm{m}$ (as much or even more than water), and thus contributes importantly to spectra. The variation in the other parameters (eddy diffusion coefficient and UV flux) has almost no impact on the emission spectra, except for the case $\zeta_{1} T_{+100}$ (reddish curves). Between 10 and $11 \mu \mathrm{m}$, there are differences in the brightness temperature of about $10 \mathrm{~K}$ due to the change of ammonia abundance. Between 13.5 and $14 \mu \mathrm{m}$, the small variations of brightness temperature are attributed to $\mathrm{HCN}$ and $\mathrm{NH}_{3}$, which both contribute strongly to the spectra in this wavelength region. Nevertheless, the differences from one spectra to another, due only to the change of eddy diffusion coefficient and UV flux, are very small, and probably not detectable with our current technologies (e.g., Stevenson et al. 2010) that do not obtain 


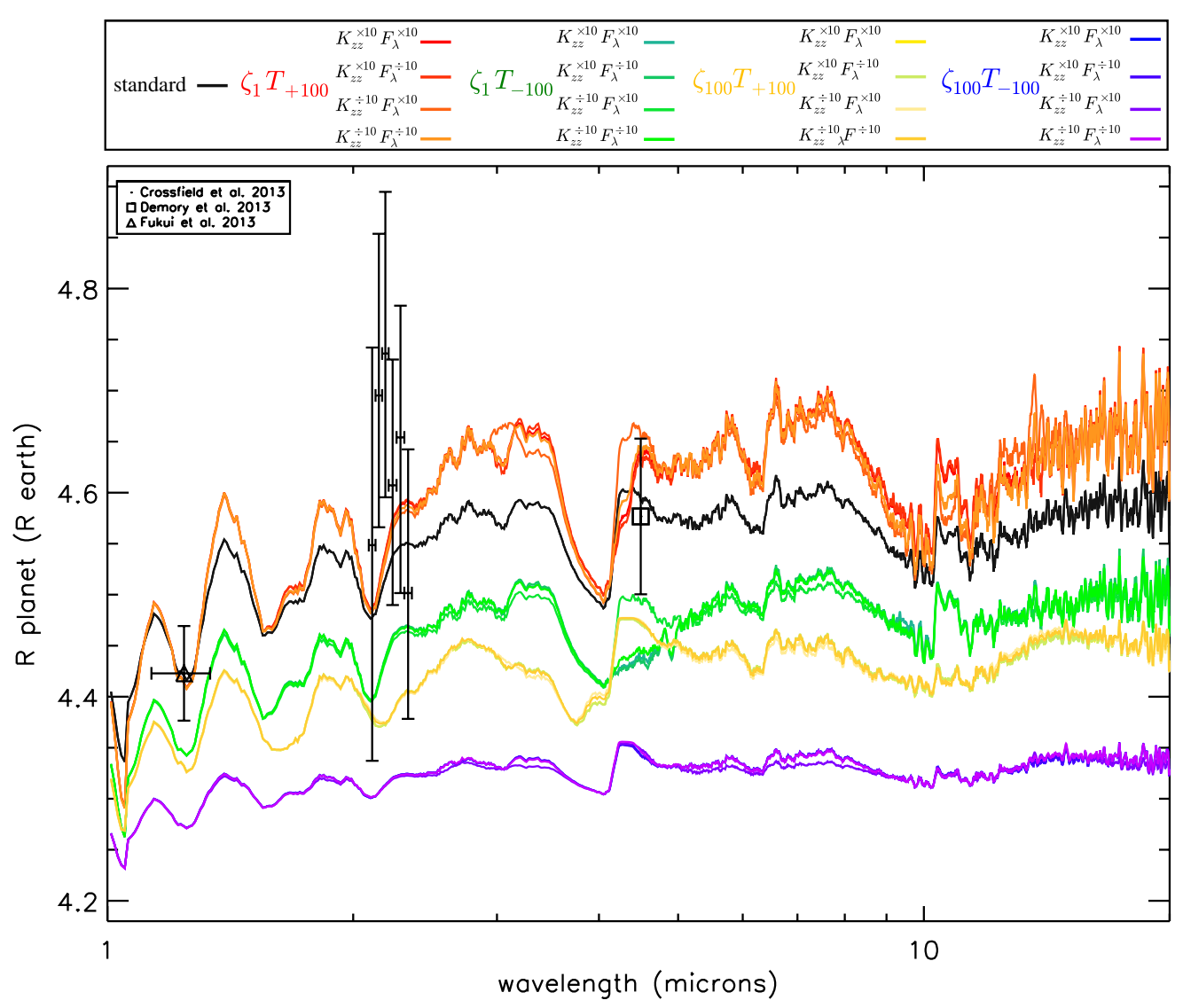

Fig. 8. Synthetic transmission spectra of GJ $3470 \mathrm{~b}$ in terms of apparent planetary radius, which are computed for all the 16 models of our grid and the standard model. Each colour corresponds to a set of metallicity and thermal profile. A colour gradient is then used to differentiate the eddy diffusion coefficients and stellar UV fluxes (see legend in the top panel and meaning of each symbol in Table 2). The standard values are shown in Figs. 2 and 3. The standard metallicity is $10 \times$ solar $(\zeta=10)$. Observational data points (references in the legend) have also been plotted for comparison. uncertainties lower than 20 K for GJ 436b with the Spitzer Space Telescope.

The transmission spectra are also separated depending on the temperature and the metallicity. Nevertheless, we notice that the greenish and yellowish spectra (respectively $\zeta_{1} T_{-100}$ and $\left.\zeta_{100} T_{+100}\right)$ are close and intersect between 4 and $5 \mu \mathrm{m}$, although the chemical composition corresponding to these 8 cases are different. The apparent planetary radius found with our synthetic spectra goes from 4.25 to $4.75 R_{\oplus}$. It is important to keep in mind that these numerical values depend on the choice of the radius of the planet at the 1 bar pressure level. Indeed, the observations give only the apparent radius of the planet, and we cannot know to which pressure level it corresponds. Changing the radius at the 1 bar pressure level translates the spectra vertically. What is important to study is the relative variation in spectra from one model to another. The radius at 1 bar is a parameter than can be adjusted to fit the observations. We decided to put the 1 bar pressure level at $4.28 R_{\oplus}$, which corresponds to the minimum apparent radius observed (Demory et al. 2013), and slightly adjusted it to fit the maximum of observational data points (Demory et al. 2013; Fukui et al. 2013; Crossfield et al. 2013) with the $\zeta_{1} T_{+100}$ and standard models. With a higher radius at $1 \mathrm{bar}$, the $\zeta_{1} T_{-100}$ and $\zeta_{100} T_{+100}$ (greenish and yellowish curves respectively) can also fit most of the observations. In contrast, we see that the last case $\left(\zeta_{100} T_{-100}\right)$ is too flat to be in the error bars. None of the models can perfectly match all the data points of Crossfield et al. (2013).

The higher radius is found with the model $\zeta_{1} T_{+100}$ (reddish curves), because the mean molecular weight is low (as opposed to the high metallicity cases), resulting in a higher atmospheric scale height and thus in a higher radius. Compared to the yellowish curves, we see an increase up to $0.2 R_{\oplus}$. On the other hand, the smaller radius is found with the model $\zeta_{100} T_{-100}$ (blueish curves) because of the low atmospheric scale height due to the high mean molecular weight $(4.1 \mathrm{~g} / \mathrm{mole})$. We see that the 17 transmission spectra globally exhibit the same features, with a given apparent planetary radius. Transmission spectra probe an upper part of the atmosphere (with a lower temperature) as compared with emission spectra and are thus more sensitive to UV photolysis and vertical mixing. For a given $\zeta-T$ case, we can observe several variations on the spectral features. Around $3.3 \mu \mathrm{m}$ and between 7 and $9 \mu \mathrm{m}$, we clearly see that the $\mathrm{CH}_{4}$ features change from one model to an other. Between 4 and $5 \mu \mathrm{m}$, we see that the contribution of $\mathrm{CO}$ and $\mathrm{CO}_{2}$ evolve for the low metallicity cases only. It is consistent in the abundances of these two species, which almost does not change for the high metallicity cases (see Fig. 5). Finally, the $\mathrm{NH}_{3}$ feature around $10 \mu \mathrm{m}$ changes in the $\zeta_{1} T_{+100}$ case because the abundance of ammonia changes significantly with different $K_{z z}$ and $F_{\lambda}$ for this cases (see Fig. 5). These variations are small (less than $0.1 R_{\oplus}$ ) but could be detectable with current observational technologies. The Okayama Astrophysical Observatory (Fukui et al. 2013) and the Hubble Space Telescope (Pont et al. 2009, for the planet GJ 436b) for instance, are able to give error bars of only $0.1 R_{\oplus}$. A detailed study as in Tessenyi et al. (2013) applied to EChO and Shabram et al. (2011) with the JWST with regards to the capacities of these future telescopes to differentiate our models, is beyond the scope of this paper but will be the subject of a follow-up study.

Although this is the only case with $[\mathrm{CO}]>\left[\mathrm{CH}_{4}\right]$, the $\zeta_{100} T_{+100}$ spectra do not show strong features due to this different $\mathrm{CH}_{4} / \mathrm{CO}$ ratio, except that the 3.3-to-4.7 $\mu$ m radius ratio is very low on the transmission spectra. Observations at these wavelengths could thus prove useful as a diagnostic of the atmospheric carbon chemistry. 


\section{Summary and discussion}

We studied the atmospheric composition of GJ 3470b, a warm Neptune that is a promising target for spectral characterisation. To prepare and to predict these future observations, we explored the parameters that are uncertain (metallicity, vertical mixing, temperature of the atmosphere, and UV flux of the parent star) and computed 17 models. They allowed us to frame the different compositions that are possible for this planet. In most cases, the $\mathrm{CH}_{4} / \mathrm{CO}$ ratio is above 1 , although we found that carbon monoxide becomes more abundant than methane under plausible conditions. This can happen for the highest metallicity tested (100× solar), which can be expected for planets with a similar bulk composition as Uranus and Neptune; we found that some models (with a high atmospheric temperature) lead to a $\mathrm{CH}_{4} / \mathrm{CO}$ ratio under unity down to a value of 0.04 in the $10^{-4}-1$ bar pressure range in this case. We did not explore hotter temperature profiles because there is no reason to get a higher temperature for a given irradiation without a significant increase of the internal heat source (similar to what has been done by Agúndez et al. 2014 for GJ 436b). It has already been shown with hot Jupiters that a higher temperature leads to a $\mathrm{CH}_{4} / \mathrm{CO}$ ratio that is lower than 1 (e.g. Moses et al. 2011; Venot et al. 2012). Moreover, our goal is not to map all the possible ranges of temperature, vertical mixing, and metallicity that can produce a $\mathrm{CH}_{4} / \mathrm{CO}$ ratio lower than unity but to address how to get it. Because of similar physical properties, this result can be extrapolated to other warm (sub-)Neptunes, such as GJ 436b or GJ 1214b. Recently, a similar study has been carried out by Moses et al. (2013), who also find that a high metallicity could lead to a $\mathrm{CH}_{4} / \mathrm{CO}$ ratio lower than 1 in GJ 436b. While the identification of the C-bearing species from observations is still under debate for these kind of planets (Stevenson et al. 2010; Madhusudhan \& Seager 2011; Beaulieu et al. 2011, with GJ 436b), these results show that the situation is not simple even from a chemical model point of view. $\mathrm{CH}_{4}$ may or may not be the major carbon reservoir, depending on the metallicity, the temperature, and the vertical mixing. Indeed, we show in this paper that there is a combined effect of these parameters on the chemical composition of atmospheres. Because of quenching, the composition of the middle atmosphere can be affected by temperatures found much deeper than the observations. This carbon anomaly depends on the temperature contrast between the probed layers, the quenching level, and the efficiency of the vertical mixing. At a metallicity higher than $100 \times$ solar, the vertical mixing can propagate a $\mathrm{CO} / \mathrm{CH}_{4}$ ratio above unity to the upper layers of the atmospheres. To retrieve the elemental abundances of such atmospheres, self-consistent models that couple all these influences are needed. Nevertheless, a very high metallicity ( $\geq 100$ times solar metallicity) seems to be a solution to explore in order to interpret future observations, as it is very likely for these atmospheres. The synthetic spectra we computed indicate that the brightness temperature as well as the transit depth vary significantly with the metallicity and the thermal profile, so future observations of GJ 3470 b may be able to determine the metallicity and the temperature of this planet. Because of the strong opacities, spectra corresponding to high metallicity models $(100 \times$ solar $)$ produce smaller features than low metallicity models $(1 \times$ solar $)$. On the spectra corresponding to the primary transit, we found that the 3.3-to- $4.7 \mu \mathrm{m}$ ratio changes together with the $\mathrm{CO} / \mathrm{CH}_{4}$ ratio. Observations at these wavelengths are a possible way to constrain this ratio.

Acknowledgements. O.V. acknowledges support from the KU Leuven IDO project IDO/10/2013 and from the FWO Postdoctoral Fellowship programme. M.A., and F.S. acknowledge support from the European Research Council (ERC
Grant 209622: E 3 ARTHs). Computer time for this study was provided by the computing facilities MCIA (Mésocentre de Calcul Intensif Aquitain) of the Université de Bordeaux and of the Université de Pau et des Pays de l'Adour.

\section{References}

Agúndez, M., Venot, O., Iro, N., et al. 2012, A\&A, 548, A73 Agúndez, M., Venot, O., Selsis, F., \& Iro, N. 2014, ApJ, 781, 68

Alibert, Y., Mordasini, C., Benz, W., \& Winisdoerffer, C. 2005, A\&A, 434, 343 Asplund, M., Grevesse, N., Sauval, A. J., \& Scott, P. 2009, ARA\&A, 47, 481 Baraffe, I., Chabrier, G., \& Barman, T. 2008, A\&A, 482, 315

Barber, R., Tennyson, J., Harris, G., \& Tolchenov, R. 2006, MNRAS, 368, 1087 Bean, J. L., Kempton, E. M.-R., \& Homeier, D. 2010, Nature, 468, 669 Bean, J. L., Désert, J.-M., Kabath, P., et al. 2011, ApJ, 743, 92 Beaulieu, J.-P., Tinetti, G., Kipping, D. M., et al. 2011, ApJ, 731, 16 Berta, Z. K., Charbonneau, D., Désert, J.-M., et al. 2012, ApJ, 747, 35 Bonfils, X., Gillon, M., Udry, S., et al. 2012, A\&A, 546, A27 Bonfils, X., Delfosse, X., Udry, S., et al. 2013, A\&A, 549, A109 Croll, B., Albert, L., Jayawardhana, R., et al. 2011, ApJ, 736, 78 Crossfield, I., Barman, T., \& Hansen, B. M. 2011, ApJ, 736, 132 Crossfield, I. J. M., Barman, T., Hansen, B. M. S., \& Howard, A. W. 2013, A\&A, 559, A33

de Mooij, E. J. W., Brogi, M., de Kok, R. J., et al. 2012, A\&A, 538, A46

Demory, B.-O., Torres, G., Neves, V., et al. 2013, ApJ, 768, 154

Désert, J.-M., Bean, J., Miller-Ricci Kempton, E., et al. 2011, ApJ, 731, L40

Elkins-Tanton, L. T., \& Seager, S. 2008, ApJ, 685, 1237

France, K., Froning, C. S., Linsky, J. L., et al. 2013, ApJ, 763, 149

Fukui, A., Narita, N., Kurosaki, K., et al. 2013, ApJ, 770, 95

Grillmair, C., Burrows, A., Charbonneau, D., et al. 2008, Nature, 456, 767 Gueymard, C. 2004, Sol. Energy, 76, 423

Harpsøe, K. B. W., Hardis, S., Hinse, T. C., et al. 2013, A\&A, 549, A10 Hauschildt, P., Allard, F., \& Baron, E. 1999, ApJ, 512, 377

Hersant, F., Gautier, D., \& Lunine, J. 2004, Planet. Space Sci., 52, 623

Hollis, M., Tessenyi, M., \& Tinetti, G. 2013, Comput. Phys. Commun. Iro, N., Bézard, B., \& Guillot, T. 2005, A\&A, 436, 719

Johnson, J. A., Butler, R. P., Marcy, G. W., et al. 2007, ApJ, 670, 833

Knutson, H. A., Madhusudhan, N., Cowan, N. B., et al. 2011, ApJ, 735, 27

Kopparapu, R. K., Kasting, J. F., \& Zahnle, K. J. 2012, ApJ, 745, 77

Lewis, J. S., \& Fegley Jr, M. B. 1984, Space Sci. Rev., 39, 163

Lewis, N. K., Showman, A. P., Fortney, J. J., et al. 2010, ApJ, 720, 344

Liang, M., Parkinson, C., Lee, A., Yung, Y., \& Seager, S. 2003, ApJ, 596, L247

Liang, M., Seager, S., Parkinson, C., Lee, A., \& Yung, Y. 2004, ApJ, 605, L61

Line, M., Liang, M., \& Yung, Y. 2010, ApJ, 717, 496

Line, M., Vasisht, G., Chen, P., Angerhausen, D., \& Yung, Y. 2011, ApJ, 738, 32

Madhusudhan, N., \& Seager, S. 2009, ApJ, 707, 24

Madhusudhan, N., \& Seager, S. 2011, ApJ, 729, 41

Miller-Ricci Kempton, E., Zahnle, K., \& Fortney, J. J. 2012, ApJ, 745, 3

Moses, J., Visscher, C., Fortney, J., et al. 2011, ApJ, 737, 15

Moses, J. I., Line, M. R., Visscher, C., et al. 2013, ApJ, 777, 34

Nascimbeni, V., Piotto, G., Pagano, I., et al. 2013, A\&A, 559, A32

Parmentier, V., Showman, A. P., \& Lian, Y. 2013, A\&A, 558, A91

Pollack, J. B., Hubickyj, O., Bodenheimer, P., et al. 1996, Icarus, 124, 62

Pont, F., Gilliland, R., Knutson, H., Holman, M., \& Charbonneau, D. 2009, MNRAS, 393, L6

Prinn, R. G., \& Barshay, S. S. 1977, Science, 198, 1031

Rothman, L., Gordon, I., Barbe, A., et al. 2009, J. Quant. Spec. Radiat. Transf., 110,533

Rothman, L., Gordon, I., Barber, R., et al. 2010, J. Quant. Spec. Radiat. Transf., 111,2139

Segura, A., Kasting, J., Meadows, V., et al. 2005, Astrobiol., 5, 706

Shabram, M., Fortney, J. J., Greene, T. P., \& Freedman, R. S. 2011, ApJ, 727, 65

Southworth, J. 2010, MNRAS, 408, 1689

Stevenson, K., Harrington, J., Nymeyer, S., et al. 2010, Nature, 464, 1161

Swain, M., Vasisht, G., \& Tinetti, G. 2008, Nature, 452, 329

Swain, M., Tinetti, G., Vasisht, G., et al. 2009a, ApJ, 704, 1616

Swain, M., Vasisht, G., Tinetti, G., et al. 2009b, ApJ, 690, L114

Tessenyi, M., Tinetti, G., Savini, G., \& Pascale, E. 2013, Icarus, 226, 1654

Tinetti, G., Meadows, V. S., Crisp, D., et al. 2005, Astrobiol., 5, 461

Tinetti, G., Meadows, V. S., Crisp, D., et al. 2006, Astrobiol., 6, 34

Tinetti, G., Vidal-Madjar, A., Liang, M., et al. 2007, Nature, 448, 169

Venot, O., Hébrard, E., Agúndez, M., et al. 2012, A\&A, 546, A43

Visscher, C., \& Moses, J. I. 2011, ApJ, 738, 72

Zahnle, K., Mac Low, M.-M., Lodders, K., \& Fegley, B. 1995, Geophys. Res. Lett., 22, 1593

Zahnle, K., Marley, M., Freedman, R., Lodders, K., \& Fortney, J. 2009a, ApJ, 701, L20

Zahnle, K., Marley, M. S., \& Fortney, J. J. 2009b, ApJ, submitted [arXiv:0911.0728] 